\title{
A Taxonomia de Bloom Revisada como suporte para o planejamento de uma disciplina de Redes de Computadores
}

\author{
Otacílio José Pereira1, Rossini Pena Abrantes', \\ Filipe Arantes Fernandes ${ }^{1}$, Loham Santos da Silva ${ }^{1}$ \\ ${ }^{1}$ Campus Manhuaçu - Instituto Federal do Sudeste de Minas Gerais (IFSudesteMG) \\ Rodovia BR 116, Km 589,8 - 36905-000 - Manhuaçu - MG - Brazil \\ \{otacilio.pereira, rossini.abrantes, \\ filipe.arantes, loham.silva\} Aifsudestemg.edu.br
}

\begin{abstract}
In this report, the planning of a discipline of computers networks is done with the decomposition into its chapters and topics and subsequent classification of each topic according to Bloom's Taxonomy Revised. This generated visions and reflections that positioned the topics according to the level of difficulty and also stimulated the search for other pedagogical strategies. This procedure was important to deal with some challenges: the adaptation of the teacher to high school, the beginning in a new institution and the offer of the discipline in the Emergency Remote Teaching model due to the pandemic. The results demonstrated the usefulness of taxonomy for the management and conduct of the discipline.
\end{abstract}

Resumo. Neste relato o planejamento de uma disciplina de redes de computadores é feito com a decomposição em seus capítulos e tópicos e posterior classificação de cada tópico conforme a Taxonomia de Bloom Revisada. Isso gerou visões e reflexões que posicionaram os tópicos conforme o nível de dificuldade e ainda estimulou a busca por outras estratégias pedagógicas. Esta maior sistemática foi importante para lidar com alguns desafios: a adaptação do professor ao ensino médio, o início em uma nova instituição e a oferta da disciplina em modelo de Ensino Retomo Emergencial (ERE) por conta da pandemia. Os resultados demonstraram a utilidade da taxonomia para essa gestão e condução da disciplina.

\section{Introdução}

O planejamento de uma disciplina é importante para prever boas estratégias para a sua condução. Ao assumir um componente curricular o professor segue um processo de compreender a ementa, refinar objetivos, estruturar conteúdo programático e imaginar estratégias pedagógicas e avaliações. Em geral isso é influenciado pelas crenças e experiências passadas do professor. Apesar de já prover resultados satisfatórios, há uma certa subjetividade e eventuais incertezas nas decisões, por isso é interessante buscar instrumentos que apoiem esta atividade para uma melhor gestão do professor.

O objetivo deste trabalho é apresentar um relato sobre a forma como a Taxonomia de Bloom, sobretudo a sua versão revisada, contribuiu para um professor planejar e se preparar para uma disciplina de Redes de Computadores. $\mathrm{O}$ foco foi buscar instrumentos que permitissem decisões mais bem refletidas, com fatores e premissas melhores investigados e com o propósito de mitigar riscos diante de alguns desafios. 
A busca por instrumentos de apoio para obter um melhor planejamento já seria uma boa motivação em circunstâncias normais, no contexto deste relato se tornou ainda mais relevante por conta de alguns desafios. Uma primeira situação foi que o professor estava iniciando em uma nova instituição, um instituto federal, em uma região diferente e em um curso técnico de nível médio. Antes o professor atuava em outra região do país no ensino superior. Além disso o professor há algum tempo não lida com o assunto de redes de computadores o que demandava uma reciclagem consistente. Por fim, um agravante foi a oferta da disciplina em formato de Ensino Remoto Emergencial, por conta da pandemia. Conforme Hodges et al. (2020), "Ensino Remoto Emergencial (ERE) é uma mudança temporária para um modo de ensino alternativo devido a circunstâncias de uma crise como guerras, desastres naturais e epidemias". Enfim, este cenário justificava a busca por uma sistemática de planejamento mais reflexiva e prudente do que em situações normais.

A seção seguinte apresenta uma revisão da bibliografia sobre Taxonomia de Bloom e sobre simuladores, uma estratégia usada na disciplina. O cenário de aplicação é comentado na seção 3 e na sequência, na seção 4, discute-se o emprego da taxonomia. A seção 5 trata os resultados com base nos dados levantados e, por fim, a seção 6 apresenta as considerações finais.

\section{Revisão bibliográfica}

Para compreensão do trabalho vale explorar dois assuntos usados como base: a Taxonomia de Bloom e o uso de simuladores no ensino de redes de computadores.

\subsection{Taxonomia de Bloom}

Segundo Krathwohl (2002), a Taxonomia de Bloom é uma estrutura ou referencial (framework) para classificar afirmações ou frases conforme o que se espera do aprendizado de alunos em um processo de instrução. Em Lima (2009) comenta-se que esta estrutura de classificação nasceu de discussões em uma reunião de examinadores universitários em 1948 e depois, em 1956, foi publicado um primeiro relatório. Apesar do nome de Benjamin $\mathrm{S}$. Bloom ser mais difundido vários especialistas foram envolvidos e eles buscavam uma estrutura que facilitasse a comunicação entre examinadores e proporcionasse a troca de ideias e materiais como questões em provas. A forma encontrada para esta estrutura foi um sistema de classificação de objetivos educacionais, uma vez que estes constituem a base do planejamento do currículo e da avaliação além de serem o ponto de partida para pesquisas educacionais (Santos, 2016).

Esta taxonomia é muito disseminada e em seu domínio cognitivo ela é organizada em seis categorias relacionadas com o ato de aprender, são elas: conhecimento, compreensão, aplicação, análise, síntese e avaliação. A taxonomia ainda estabelece uma relação de dependência, pressupõe que para atingir uma categoria os objetivos e categorias anteriores precisam ser dominados. Cada categoria possui alguns verbos associados para facilitar a classificação dos objetivos pretendidos. Para exemplificar, a categoria "conhecimento" está associada a verbos como nomear, rotular e declarar e a categoria "aplicação", a verbos como empregar, operar, manipular e usar. 


\subsection{Taxonomia de Bloom Revisada}

A taxonomia passou por um processo de revisão anos depois. Segundo Marchesan e Kuhn (2018) especialistas se reuniram em Nova Iorque para rever os pressupostos uma vez que a esfera educacional contava com novas teorias, conceitos e recursos. Daí, em 2010, foi publicado um relatório desta revisão supervisionada por David Krathwohl.

Uma mudança substancial foi obter uma estrutura com duas dimensões. A dimensão do processo cognitivo foi mantida com as 6 categorias que passam a ser rotuladas com verbos: lembrar, entender, aplicar, analisar, avaliar e criar (sintetizar). Além disso observa-se que houve a troca de nome de "síntese" para "criar" e a inversão de ordem entre "criar" (síntese) e "avaliar" (Gil, 2015). Uma nova dimensão foi adicionada, a que trata o tipo do conhecimento com 4 categorias: o conhecimento efetivo/factual que diz respeito a terminologia e elementos específicos; o conceitual que já envolve categorizações, modelos e estruturas; o procedural que trata aspectos sobre como fazer e por fim a categoria mais avançada, o conhecimento metacognitivo sobre estratégias e estilos para a aprendizagem (Marchesan e Kuhn, 2018). O Quadro 2 mostra como esta taxonomia pode ser disposta como tabela bidimensional, base para posicionar os objetivos educacionais como foi feito nesse relato.

Entender essas taxonomias tem a sua importância pois o uso em um processo educacional apresenta benefícios. Ferraz e Belhot (2010) apresenta que elas ajudam a desenvolver os instrumentos de avaliação, auxiliam no uso de estratégias para facilitar e estimular o desempenho dos alunos em diferentes níveis de aquisição de conhecimento e ainda estimula educadores a auxiliarem seus discentes de forma estruturada e consciente. Vários trabalhos apresentam a forma como a taxonomia de Bloom pode ser usada. Em Dargains e Sampaio (2020) ela auxilia na concepção de estratégias para melhorar a qualidade do ensino de programação introdutória e pensar caminhos para o ensino de ciência e tecnologia em escolas públicas. Já Barreto et al. (2019) usa para conceber instrumentos de avaliação em uma criação de um objeto de aprendizagem para ensino de pegada ecológica. Santos (2016) aplica na concepção de uma plataforma e ferramenta para guiar professores no planejamento de cursos MOOC.

Marchesan e Kuhn (2018) apresenta uma proposta muito interessante do uso da taxonomia em um curso de administração em que os objetivos educacionais de disciplinas são posicionados na estrutura bidimensional da TBR permitindo uma análise dos propósitos do curso e eventuais reformulações. Essa abordagem inspirou o uso no planejamento da disciplina desse relato, se lá a decomposição e análise foi no contexto curso-disciplinas-objetivos, aqui a quebra foi em disciplina-capítulos-tópicos-objetivos.

\subsection{O uso de simuladores no ensino de redes de computadores}

Algumas reflexões provocadas ao aplicar a taxonomia nos tópicos da disciplina permitiram pensar alternativas para contornar a adoção do ensino remoto e simuladores para o ensino de rede foi um dos caminhos imaginados. Conforme Silva et al. (2019) estes softwares são usados sobretudo quando há limitações em infraestrutura de laboratórios e alguns exemplos são: CORE, NetKit, OMNet++ e Cisco Packet Tracer.

A opção foi pelo Cisco Packet Tracer por ser oferecido gratuitamente pela Cisco, uma empresa reconhecida na área pelos seus equipamentos e pelo seu programa de certificação profissional (Cisco Networking Academy, 2021). Em Silva et al. (2019) esse simulador foi experimentado com bons resultados mas com ressalvas quanto à 
interface que poderia ser mais simples. De fato, por ser muito completo, esse simulador exige mais do usuário seja pelos vários tipos de equipamentos e janelas, seja pelos comandos e outros recursos que oferece. Para o ambiente desse relato havia uma preocupação quanto ao nível dos alunos para acomodar a tecnologia, sobretudo remotamente. Em uma nova pesquisa encontrou-se uma versão mais simples, o PT (Packet Tracer) Anywhere (Figura 1), com interface enxuta e intuitiva e na Web (online), portanto sem necessidade de instalação (Mikroyannidis et al., 2018).

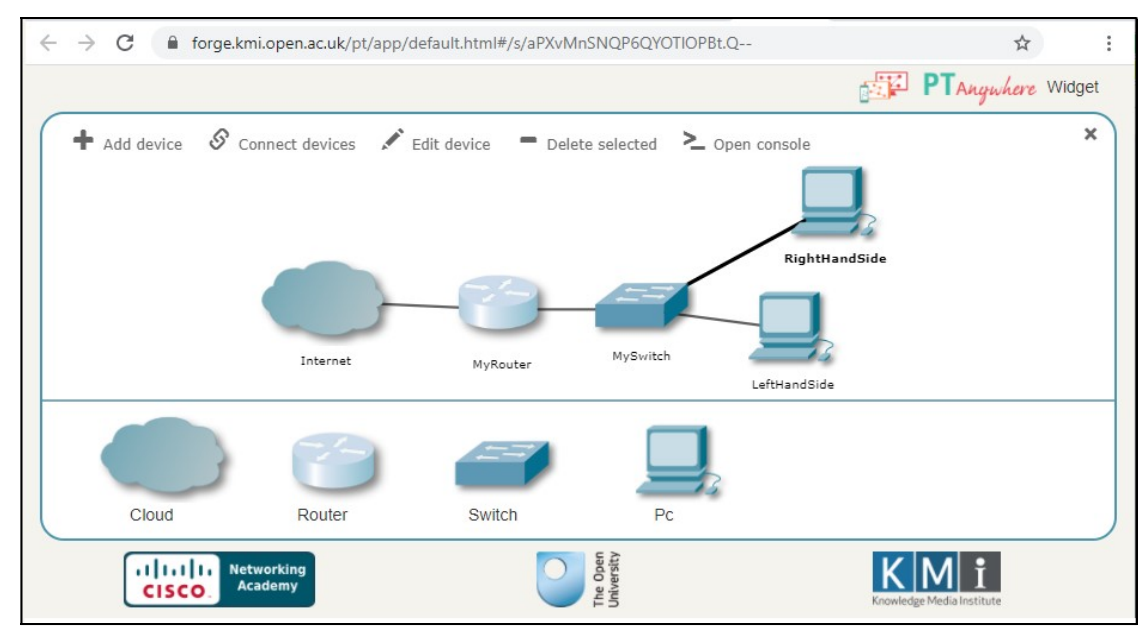

Figura 1. Simulador PT (Packet Tracer) Anywhere (Fonte: PT Anywhere, 2021)

\section{Cenário de aplicação}

Essa experiência ocorreu em uma disciplina de Redes de Computadores I de um curso técnico em informática concomitante ou subsequente de um instituto federal localizado no interior do sudeste de Minas Gerais. A ementa da disciplina explora a introdução à ao assunto, tipos de rede, topologias, protocolos, modelo OSI e TCP/IP, interconexão de redes, endereçamento IP, cabeamento e configuração de equipamentos. O objetivo da disciplina é apresentar alguns aspectos teóricos, o funcionamento básico $\mathrm{e}$ procedimentos de montagem de uma pequena rede.

Por conta da pandemia, apenas parte da disciplina (50\%) foi ofertada no regime do ERE e, da ementa, trataram-se assuntos teóricos e aspectos práticos independentes da infraestrutura física como configurações lógicas nos simuladores. Depois, com o retorno das aulas presenciais, ela será complementada com as práticas em laboratório com os equipamentos físicos. Na implantação do ERE foi verificado se os alunos teriam acesso a computador e internet e um programa de assistência para aquisição desses recursos foi realizado. Assim, a turma com 10 alunos, alguns de zona rural, podiam acompanhar as aulas pela Internet sem a necessidade, por exemplo, de recursos impressos.

\section{Emprego da Taxonomia de Bloom no planejamento da disciplina}

O professor já tinha conhecimento da versão inicial da Taxonomia de Bloom por conta de capacitações passadas. No ingresso na nova instituição, participando de grupos de estudos, ele conheceu a Taxonomia de Bloom Revisada e outras aplicações e daí surgiu a ideia de usá-la em seu planejamento de ensino explorando alguns benefícios. 


\subsection{Estruturações de capítulos e tópicos como "mapas de navegação"}

Pelo conhecimento prévio da versão inicial da taxonomia, o professor já tinha certa noção sobre pensar estruturações, segmentar assuntos em "mapas de navegação", seja para seus próprios estudos, seja para guiar o aprendizado de alunos. Desta forma, uma primeira estratégia foi encontrar estruturas que permitissem uma boa visão da disciplina.

Três visões compuseram essa abordagem de estruturar a disciplina e estão mostradas na Figura 2. A primeira visão é comum em redes de computadores, trata-se da organização em camadas e apesar do modelo OSI (Open System Interconnection) ter sido estudado optou-se pelas camadas da família de protocolos TCP/IP. Essa visão de camadas está mais à esquerda (letra $\mathrm{A}$ ) com as camadas próximas dos equipamentos. $\mathrm{A}$ segunda estrutura (letra B) foca os elementos que compõem uma rede típica, por exemplo, computadores, cabos, equipamentos de rede e outros, é uma foto de uma rede física. As visões A e B se entrelaçam, pois em A é usado um esquema de rede do simulador que serve tanto para posicionar as camadas como para fornecer uma visão abstrata de rede mostrada em B via foto em uma das visitas do professor ao laboratório. A terceira visão (letra $C$ ) trata os tipos de tópicos explorados em cada um dos capítulos: contextualização, conceitos, procedimento sobre como fazer e as práticas.

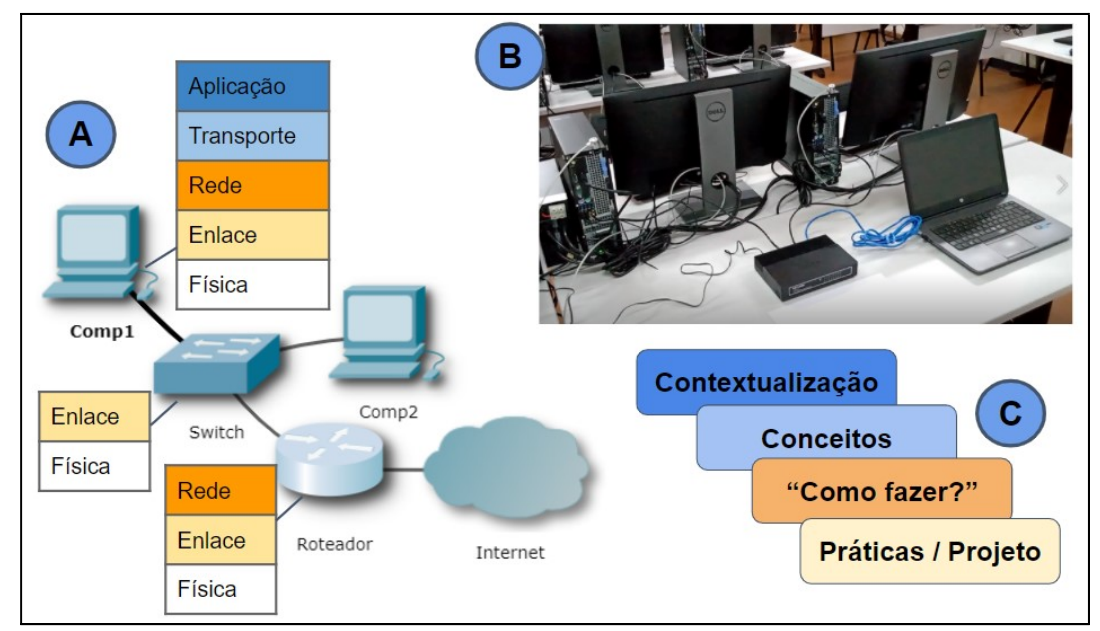

Figura 2. Estruturações usadas na disciplina (Fonte: própria)

Imagina-se que esse exercício de compor e visualizar estas estruturas exploram os objetivos educacionais ligados à análise e síntese da taxonomia por conta da imersão em ações como: decompor cada uma das visões em suas partes, compreender as partes e suas relações e de retornar a uma perspectiva macro para uma noção do todo.

\subsection{Compreensão e classificação dos tópicos conforme taxonomia}

Apesar desse uso nas estruturações já ajudar na visão da disciplina, o uso da classificação foi mais interessante quando o professor reviu a taxonomia e conheceu a Taxonomia de Bloom Revisada. Isso foi influenciado por outras iniciativas em que o professor participava, a reformulação do Projeto Pedagógico de Curso e um grupo de estudos sobre práticas pedagógicas. No grupo outro professor compartilhou o trabalho de Marchesan e Kuhn (2018) que motivou a adaptação da abordagem para esse cenário.

O exercício foi decompor a disciplina em seus capítulos e tópicos e daí fazer uma classificação com base na taxonomia. O Quando 1 apresenta o resultado em que 
cada tópico foi enquadrado nas dimensões de Processo Cognitivo (coluna 'P') e de Conhecimento em si (' $\mathrm{C}$ '). Os números indicam cada uma das categorias nas dimensões, para o processo cognitivo: (1) lembrar, (2) entender, (3) aplicar, (4) analisar, (5) avaliar e (6) criar e para a dimensão de conhecimento: (1) efetivo/factual, (2) conceitual, (3) procedural e (4) metacognitivo. Para este enquadramento, em cada tópico o professor refletia sobre os assuntos, exercícios e avaliações que seriam explorados e conforme a natureza predominante destes elementos o tópico era associado às categorias das dimensões.

Para exemplificar vale observar o tópico C4-7 de configurações IP no capítulo 4 de camada de rede. Na dimensão de processo cognitivo ('P') ele foi classificado como avaliar (5) pois além do aluno precisar de montar os elementos da rede (equivalente à categoria 4 de aplicar) ele precisava sobretudo de testar e averiguar a rede funcionando, tinha que detectar, julgar e resolver problemas e justificar suas soluções, todos esses verbos associados a categoria de avaliar (número 5). Para a dimensão conhecimento ('C') como o assunto envolve seguir procedimento para montar a rede, ainda que virtualmente em simulador, a categoria associada foi a 3 de conhecimento procedural.

Quadro 1. Classificação dos tópicos conforme taxonomia

\begin{tabular}{|l|c|c|l|c|c|}
\hline \multicolumn{1}{|c|}{ Capítulo/Tópico } & P & C & \multicolumn{1}{|c|}{ Capítulo/Tópico } & P & C \\
\hline Cap 1 - Introdução & & & Cap 4 - Camada de Rede & & \\
\hline 1) Definição e histórico & 3 & 2 & 1) Comunic. entre redes & 2 & 2 \\
\hline 2) Exemplos no mundo real & 3 & 2 & 2) Introdução protocolo IP & 2 & 2 \\
\hline 3) Tipos e topologias & 2 & 2 & 3) Endereçamento IP & 3 & 3 \\
\hline 4) Equipamentos de rede & 4 & 2 & 4) Máscara de sub-rede & 3 & 3 \\
\hline 5) Org. em camadas & 4 & 2 & 5) Gateway padrão/roteador & 2 & 2 \\
\hline 6) Observação rede do aluno & 3 & 2 & 6) DHCP & 2 & 3 \\
\hline Cap 2 - Camada Física & & & 7) Configurações IP (simulador) & 5 & 3 \\
\hline 1) Conceitos de sinais & 2 & 2 & 8) Roteamento entre rede & 2 & 2 \\
\hline 2) Modos de transmissão & 2 & 2 & 9) Rotas estáticas e dinâm (RIP) & 3 & 3 \\
\hline 3) Ethernet e IEEE 802.3 & 3 & 2 & 10)Config. Roteador (simulador) & 5 & 3 \\
\hline 4) Protocolo CSMA/CD & 4 & 2 & Cap 5 - Transp. e Aplicação & & \\
\hline 5) Tipos e categ. de cabos & 2 & 2 & 1) Introd. à camada de transporte & 2 & 2 \\
\hline 6) Padrões TIA468A e B e \\
crimpagem de cabos & 3 & 3 & 2) Serviços da camada de transp. & 2 & 2 \\
\hline Cap 3 - Camada de Enlace & & & 3) Protocolos TCP e UDP & 2 & 2 \\
\hline 1) Subcamadas LLC e MAC & 2 & 2 & 4) Introd. à camada de aplicação & 2 & 2 \\
\hline 2) Quadro Ethernet & 2 & 2 & 5) Exemplos: HTTP & 3 & 2 \\
\hline 3) Endereços MAC & 3 & 2 & 6) Exemplos: SMTP & 3 & 2 \\
\hline 4) Verificação de erros & 3 & 3 & & \\
\hline 5) Monitoramento via Wireshark & 3 & 3 & & \\
\hline
\end{tabular}

\subsection{Reflexões derivadas no uso da taxonomia e a ideação de oportunidades}

Se de início o propósito da decomposição e classificação dos tópicos era posicionar o nível de dificuldade da disciplina, ao mergulhar nesse planejamento percebia-se o surgimento de diversas reflexões derivadas que acabavam estimulando a concepção de novas alternativas pedagógicas para os tópicos.

Para exemplificar, vale selecionar tópicos em que isso foi mais significativo, situações que envolviam prática e alguma dependência da infraestrutura física. Por exemplo, no tópico de definição de rede que em laboratório seria fácil mostrar o cenário real, surgiu a ideia do aluno explorar algumas estruturas e equipamentos da sua própria 
conexão com a Internet. Uma atividade solicitava que o aluno tirasse fotos e explicasse o que era cada parte ou equipamento e a forma como eles compunham a sua rede local. Além disso ele usava comandos como "ipconfig", "ping" e "tracert" para observar configurações, testar conexão IP e traçar rotas, respectivamente. Com isso, o tópico em vez de se limitar a compreender um conhecimento factual com mera definição foi explorado com investigação da rede local sendo portanto classificado na categoria de aplicação e de conhecimento conceitual.

Outro ponto ainda mais significativo foi quando surgiu a ideia de usar simuladores para suprir as práticas laboratoriais. Por exemplo, na camada de rede um dos objetivos envolvem compreender números IP's, sua configuração e funcionamento em uma rede, vide exemplo C4-7 usado ao explicar o Quadro 1. Em um laboratório físico a predominância dos objetivos se enquadram como aplicar ou avaliar um conhecimento procedural. Com o ERE, a primeira ideia seria mostrar e discutir vídeos e isso classificaria o item como um compreender-procedural. Com o simulador abriu-se a perspectivas de objetivos mais avançados e como foi exemplificado junto ao Quadro 1, esse item C4-7 evoluiu para a categoria de avaliar (5) de conhecimento procedural (3).

Além do Quadro 1, uma outra forma de dispor essas classificações está mostrada na Quadro 2 e ele é interessante pois ilustra o que as reflexões derivadas visavam provocar, no caso o deslocamento dos tópicos para categorias mais à direita e em linhas inferiores. Pelo mapa percebe-se que pelo caráter mais teórico e introdutório, mesmo tentando ampliar os objetivos, boa parte dos tópicos concentram-se na "célula" compreender-conceitual. Entretanto, vale destacar o posicionamento dos tópicos que envolvem o simulador (C4-7 e C4-10) em "células mais avançadas".

Quadro 2. Posicionamento de tópicos na estrutura bidimensional da taxonomia

\begin{tabular}{|l|l|l|l|l|l|l|}
\hline & $\begin{array}{c}\mathbf{1 .} \\
\text { Conh. }\end{array}$ & \multicolumn{1}{|c|}{$\begin{array}{c}\mathbf{2 .} \\
\text { Compreender }\end{array}$} & $\begin{array}{c}\text { 3. } \\
\text { Aplicar }\end{array}$ & $\begin{array}{c}\text { 4. } \\
\text { Analisar }\end{array}$ & $\begin{array}{c}\mathbf{5} . \\
\text { Aval. }\end{array}$ & $\begin{array}{c}\mathbf{6 .} \\
\text { Criar }\end{array}$ \\
\hline 1.Factual & & & & & & \\
\hline 2.Conceitual & & $\mathrm{C} 1-3, \mathrm{C} 2-1, \mathrm{C} 2-2, \mathrm{C} 2-4$, & $\mathrm{C} 1-1, \mathrm{C} 1-2$, & $\mathrm{C} 1-4, \mathrm{C} 1-$ & & \\
& & $\mathrm{C} 2-5, \mathrm{C} 3-1, \mathrm{C} 3-2, \mathrm{C} 4-1$, & $\mathrm{C} 1-6, \mathrm{C} 2-3$, & 5 & & \\
& & $\mathrm{C} 4-2, \mathrm{C} 4-5, \mathrm{C} 4-8, \mathrm{C} 5-1$, & $\mathrm{C} 3-3, \mathrm{C} 5-5$, & & & \\
& & $\mathrm{C} 5-2, \mathrm{C} 5-3, \mathrm{C} 5-4$ & $\mathrm{C} 5-6$ & & \\
& & & $\mathrm{C} 2-6, \mathrm{C} 3-4$, & $\mathrm{C} 4-7, \mathrm{C} 4-$ & & \\
& & & $\mathrm{C} 4-9$ & & & \\
\hline 3.Procedural & & & & & & \\
\hline
\end{tabular}

\section{Análise de resultados e discussões}

Como um primeiro resultado vale citar a construção dos quadros discutidos anteriormente e sobretudo as decisões e as estratégias pedagógicas derivadas na construção deles. Observar e analisar os quadros permitiu refletir sobre o posicionamento predominante que se pretendia na disciplina e também decisões sobre como sequenciar e encadear os tópicos de forma a refletir a "ordem crescente" dos desafios. Por exemplo, prever tópicos mais simples no início e avançar no nível de dificuldade conforme o desenvolvimento do aluno seria interessante em turma em uma nova instituição e ainda com adaptação para o formato ERE.

Para acomodar, monitorar e validar a adoção dessas estratégias e resultados, o professor previu um levantamento de dados junto aos alunos que foi especialmente útil 
para diagnosticar eventuais problemas e promover ajustes de rota. Nos primeiros capítulos, a cada aula o aluno informava o nível de dificuldade percebido. Já no capítulo em que foi inserido o simulador, perguntas inspiradas em testes de usabilidade de software foram adotadas.

A Figura 3 resume as respostas sobre ao nível de dificuldade nas aulas dos capítulos iniciais. $\mathrm{O}$ almejado no planejamento da disciplina era atingir um nível médio com alguns pontos mais difíceis. Percebe-se que no geral a percepção dos alunos centrou-se no nível médio. As aulas do capítulo 2 (C2-A1 e C2-A2) da camada física foram consideradas também fácil ou muito fácil o que indica que poderia se exigir mais.

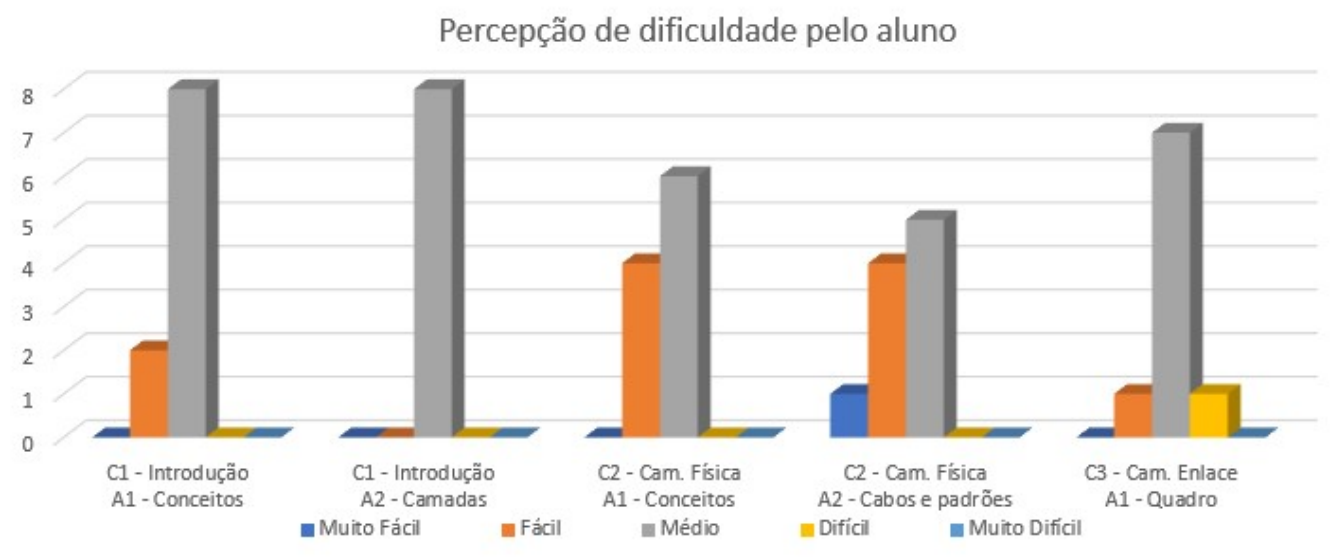

Figura 3. Percepção de dificuldade pelo aluno (Fonte: própria)

No capítulo 4 da camada de rede, em uma primeira experiência com o simulador adotou-se um levantamento por um teste de usabilidade. Para uma atividade de "montar" e configurar uma rede local com um switch, o aluno precisava realizar cada uma das tarefas de um roteiro e para cada uma ele registrava o nível de dificuldade. Eram previstas 7 tarefas desde uma ambientação inicial, criação da rede, configurações e testes finais. Analisando as respostas (Figura 4), percebe-se que nas tarefas iniciais (T1.T2 e T3) predominam respostas em nível fácil ou mesmo muito fácil. Já a partir da tarefa T4 com exploração da lógica de configuração e testes, as respostas predominantes são de nível médio. Pela simplicidade do simulador imaginavam-se boas respostas porém em níveis um pouco mais altos para as tarefas finais. Vale ainda destacar que apenas 7 dos 10 alunos entregaram a atividade, os outros 3 justificaram por conta de dificuldade em entender a lógica e fazer funcionar o experimento.

\section{Percepção de dificuldade na $1^{\mathrm{a}}$ experiência com o simulador}

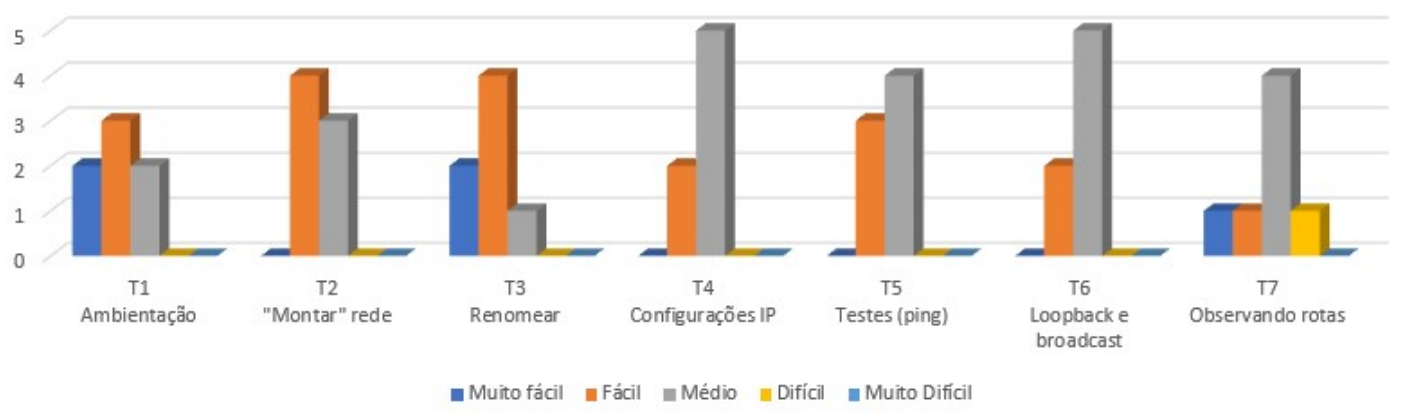

Figura 4. Percepção de dificuldade em tarefas com simulador (Fonte: própria) 
Houve uma segunda experiência com o simulador para configuração de roteamento entre duas redes. Além de montar as redes, o aluno precisava conectar e configurar um roteador entre elas. Apenas 5 alunos entregaram a atividade e eles obtiveram $61 \%$ de rendimento em média. Parte deste resultado mais baixo pode ter sido por conta da tarefa mais desafiadora, mas há também a situação conhecida de que a data de submissão do trabalho coincida com entregas de atividades de outros professores. Outra reflexão sobre esse rendimento menor é que essa parte exigia muitas tarefas com linhas de comando de configuração de roteador que exige mais do aluno.

Os últimos dados a comentar são os de desempenho nos capítulos (Quadro 3) com o percentual da nota máxima, número de entregas confrontados com dificuldade prevista pelo professor. Em cada avaliação, cada aluno tinha temas ou parâmetros diferentes para dificultar cópias de trabalhos ou outros meios não apropriados. Os capítulos iniciais, de objetivos mais simples e sem simulador, tiveram resultados melhores com mais entregas e rendimento melhor. $\mathrm{Na}$ atividade mais desafiador no capítulo 4 que envolvia roteador teve o rendimento menor e já foi discutido anteriormente. Para o capítulo 5 a atividade foi simples, uma explicação de protocolos das camadas mais altas, por isso os resultados compatíveis com os capítulos iniciais. Há certa correlação entre o rendimento e a dificuldade prevista pelo professor ao refletir a associação com a taxonomia, capítulos com categorias mais avançadas tiveram resultados mais baixos e os de objetivos simples, resultados melhores de rendimento.

Quadro 3. Desempenho e número de entregas por capítulo ou atividade

\begin{tabular}{|l|c|c|c|}
\hline \multicolumn{1}{|c|}{ Capítulo } & Des. & Entr. & Dif. Prevista \\
\hline Cap 1 - Introdução & $90 \%$ & 9 & 2 - Fácil \\
\hline Cap 2 - Camada Física & $81 \%$ & 8 & 3 - Médio \\
\hline Cap 3 - Camada de Enlace & $78 \%$ & 9 & 3 - Médio \\
\hline Cap 4 - Camada de Rede (Exp. Rede Local) & $94 \%$ & 7 & 3 - Médio \\
\hline Cap 4 - Camada de Rede (Exp. Roteamento) & $61 \%$ & 5 & 4 - Difícil \\
\hline Cap 5 - Camadas de transporte e de aplicação & $85 \%$ & 10 & 2 - Fácil \\
\hline
\end{tabular}

Uma informação adicional veio de uma reunião entre a coordenação do curso e os alunos sem a presença dos professores. $\mathrm{Na}$ ocasião os alunos de forma espontânea manifestaram identificação com as estratégias adotadas em duas disciplinas, uma delas essa de redes. Mais um indício de que as estratégias tiveram sintonia com os alunos.

\section{Considerações Finais}

O planejamento de uma disciplina envolve fatores que podem tornar a atividade subjetiva e, além disso, incertezas e dúvidas podem ocorrer por conta de circunstâncias desafiadoras do contexto. Este relato apresentou a forma como uma Taxonomia de Bloom Revisada contribuiu para um melhor planejamento e condução de uma disciplina de redes de computadores, sobretudo com sua oferta em um Ensino Remoto Emergencial. Por exemplo, classificar tópicos com a taxonomia permitiu refletir sobre estratégias pedagógicas como o uso do simulador, possibilitou identificar níveis de dificuldade de assuntos, ajudou a pensar uma melhor sequência ou mapa de exploração além de originar formas de levantar dados e avaliar a sintonia com os alunos.

Apesar de alguns resultados favoráveis é importante salientar que ainda há uma inquietação do professor quanto ao pleno domínio dos alunos sobre os assuntos e isso 
advém das dificuldades de avaliar plenamente o aluno em um formato ERE. Espera-se que na complementação da disciplina no retorno às aulas presencias, eventuais reforços e outros instrumentos sejam usados para validar e refinar o nível de aprendizado.

\section{Referências}

Barreto, M. et al. (2019). Criação e avaliação de um objeto de aprendizagem para a pegada ecológica. VIII Congresso Brasileiro de Informática na Educação (CBIE 2019).

Cisco Networking Academy (2021). Cisco Packet Tracer. Disponível em: $<$ https://www.netacad.com/pt-br/courses/packet-tracer >. Acesso em: 15 abr 2021.

Dargains, A. Sampaio, F. (2020). Estudo Exploratório sobre o Uso da Robótica Educacional no Ensino de Introdução a Programação. Tecnologias, sociedade e conhecimento, v. 7, n. 1.

Ferraz, A. Belhot, R. (2010) Taxonomia de Bloom: revisão teórica e apresentação das adequações do instrumento para definição de objetivos instrucionais. Gestão \& Produção - Gest. Prod. (Online), v.17, n.2, pp.421-431.

Gil, Antonio Carlos. Didática no ensino superior. São Paulo: Atlas, 2015. 111p.

Hodges, C. Moore, S. Lockee, B. Trust, T. Bond, A. The Difference Between Emergency Remote Teaching and Online Learning. Educause Review. Disponível em: $\quad<$ https://er.educause.edu/articles/2020/3/the-difference-between-emergencyremote-teaching-and-online-learning $>$. Acesso em: 15 jul 2020.

Krathwohl, D. R. (2002). A revision of bloom's taxonomy: An overview. Theory into practice, Taylor \& Francis, v. 41, n. 4, p. 212-218.

Lima, R. (2009) Mapa de Conteúdos e Mapa de Dependências: ferramentas pedagógicas para uma metodologia de planejamento baseada em objetivos educacionais e sua implementação em um ambiente virtual de aprendizagem. Tese (Doutorado).

Marchesam M. R. Kuhn, M. K. (2018) Análise dos objetivos educacionais de um Curso Técnico de Administração pela Taxonomia de Bloom Revisada. Cadernos de Educação - UFPel (Online).

Mikroyannidis, A., Gómez-Goiri, A., Smith, A. \& Domingue, J. (2018) PT Anywhere: a mobile environment for practical learning of network engineering. Interactive Learning Environments, 1-15.

PT Anywhere. (2021). PT Anywhere network simulation. Disponível em: $<$ https://ptanywhere.kmi.open.ac.uk/>. Acesso em: 22 fev 2021.

Santos, R. (2016) Inserindo a Taxonomia Revisada de Bloom em um MOOC. Dissertação de mestrado. Programa de Pós-Graduação em Ciência da Computação Universidade do Estado do Rio Grande do Norte e a Universidade Federal Rural do Semi-Árido.

Silva, R. et al. (2019) Uso do Cisco Packet Tracer como ferramenta no ensinoaprendizagem de Redes de Computadores no IFRN - Campus Mossoró. ScientiaTec: Revista de Educação, Ciência e Tecnologia do IFRS, v.6, n.2, p: 67-78. 